\title{
CUMPRIMENTO DA TERAPIA COM ANTIDIABÉTICOS ORAIS EM USUÁRIOS DA ATENÇÃO PRIMÁRIA ${ }^{1}$
}

\author{
Márcio Flávio Moura de Araújo², Roberto Wagner Júnior Freire de Freitas³, Luciana Vládia Carvalhêdo \\ Fragoso ${ }^{4}$, Thiago Moura de Araújo ${ }^{5}$, Marta Maria Coelho Damasceno ${ }^{6}$, Maria Lúcia Zanetti ${ }^{7}$
}

\footnotetext{
${ }^{1}$ Esta pesquisa recebeu financiamento do Conselho Nacional de Pesquisa e Desenvolvimento Tecnológico (CNPq).

${ }^{2}$ Doutorando do Programa de Pós-Graduação em Enfermagem da Universidade Federal do Ceará (UFC). Professor do Curso de Enfermagem da Universidade Federal do Maranhão-Imperatriz. Maranhão, Brasil. E-mail: marciofma@yahoo.com.br

${ }^{3}$ Mestre em Enfermagem. Professor do Curso de Enfermagem da Universidade Federal do Piauí-Floriano. Piauí, Brasil. E-mail: robertowjff@globo.com

${ }^{4}$ Mestre em Enfermagem. Enfermeira do Hospital Universitário Walter Cantídio da UFC. Ceará, Brasil. E-mail: luciana.vladia@ hotmail.com

${ }^{5}$ Doutorando do Programa de Pós-Graduação em Enfermagem da UFC. Professor do Curso de Enfermagem da Universidade Federal do Maranhão-Imperatriz. Maranhão, Brasil. E-mail: thiagomouraenf@yahoo.com.br

${ }^{6}$ Doutora em Enfermagem. Professora do Programa de Pós-Graduação em Enfermagem da UFC. Pesquisadora do CNPq. Ceará, Brasil. E-mail: martadamasceno@terra.com.br

7 Doutora em Enfermagem. Professora da Escola de Enfermagem de Ribeirão Preto da Universidade de São Paulo. São Paulo, Brasil. E-mail: zanetti@eerp.br
}

\begin{abstract}
RESUMO: O objetivo deste estudo foi avaliar o cumprimento medicamentoso de usuários de antidiabéticos orais. A pesquisa envolveu 437 diabéticos, de ambos os sexos, com idade entre 18-92 anos de 12 unidades básicas de saúde de Fortaleza-Ceará entre março e junho de 2009. Para avaliar a adesão aos antidiabéticos orais aplicou-se, nos domicílios, o Teste de Batalla, o Teste de Medida de Adesão ao Tratamento, além da contagem de comprimidos. Constatou-se, que 74,6\%,86,3\% e 71,2\%, foram considerados não cumpridores, segundo os testes aplicados, respectivamente. Entre os sujeitos não cumpridores do tratamento com antidiabéticos, $95 \%$ já faltaram as consultas do programa terapêutico para diabetes $(p=0,000)$ e $76 \%$ estão com a glicemia acima dos valores recomendados $(p=0,013)$. Talvez o cerne do problema esteja na relação profissional/cliente e na educação em saúde. Portanto, o resgate dos fundamentos da promoção da saúde necessitam permear as ações dos profissionais envolvidos na atenção ao diabético.
\end{abstract}

DESCRITORES: Cooperação do paciente. Diabetes Mellitus tipo 2. Hipoglicêmicos

\section{ORAL ANTIDIABETIC DRUG THERAPY COMPLIANCE AMONG BRAZILIAN PUBLIC HEALTH SYSTEM USERS}

\begin{abstract}
This transversal study aimed to evaluate compliance with pharmacological therapy among users of oral antidiabetic drugs. The research involved 437 diabetic patients of both sexes between 18-92 years of age from 12 healthcare centers located in Fortaleza, Ceará, Brazil, between March and June of 2009. In order to evaluate their adhesion to oral antidiabetic medication, both Batalla's Test and the Treatment Adherence and Measurement Test were applied in households. Tablets were also counted. It was established that $74.6 \%, 86.3 \%$ and $71.2 \%$ were considered non-compliers according to the tests applied, respectively. Among the subjects not complying with their treatment via oral antidiabetics, $95 \%$ have missed diabetes therapeutic program appointments $(\mathrm{p}=0.000)$ and $76 \%$ maintain blood glucose levels above recommended values $(\mathrm{p}=0.013)$. It is possible that the core of the problem is in the professional-client relationship and in health education. Therefore, the revival of the foundations of health promotion need to permeate the actions of professionals involved in diabetic patient care.
\end{abstract}

DESCRIPTORS: Patient compliance. Diabetes Mellitus type 2. Hypoglycemic agents.

\section{EL CUMPLIMIENTO DEL TRATAMIENTO CON ANTIDIABÉTICOS ORALES EN USUARIOS DE LA ATENCIÓN BÁSICA}

RESUMEN: El objetivo de este estudio fue evaluar el cumplimiento del tratamiento farmacológico por los usuarios de antidiabéticos orales. En la investigación participaron 437 pacientes diabéticos de ambos sexos, con edades entre 18 a 92 años, de 12 unidades básicas de salud de la ciudad de Fortaleza - Ceará, Brasil, de marzo a junio de 2009. Con el fin de evaluar la adhesión a los antidiabéticos orales se aplicaron en los hogares dos pruebas: el Test de Batalla y el test para medir la adhesión al tratamiento, además del recuento de pastillas. Se constató que el 74,6\%,86,3\% y 71,2\% se consideran no cumplidores del tratamiento, de acuerdo a las pruebas aplicadas, respectivamente. Entre los que no cumplen el tratamiento con antidiabéticos, el 95\% faltaron a las consultas del programa de tratamiento para la diabetes $(p=0,000)$ y el $76 \%$ presentan los niveles de glucosa en la sangre más altos que los valores recomendados $(p=0,013)$. Tal vez el núcleo del problema está en su relación profesional/cliente y en la educación para la salud. Por lo tanto, el rescate de las bases de la promoción de la salud debe impregnar la actuación de los profesionales que intervienen en el cuidado de los pacientes diabéticos.

DESCRIPTORES: Cooperación del paciente. Diabetes Mellitus tipo 2. Agentes hipoglucémicos. 


\section{INTRODUÇÃO}

A literatura destaca que, para os profissionais de saúde que cuidam de sujeitos com Diabetes Mellitus do tipo 2 (DM2), a questão mais desafiadora é o controle glicêmico. ${ }^{1-3}$ Por sua vez, o controle da glicemia guarda estreita relação com o cumprimento do tratamento prescrito, o qual abrange medidas medicamentosas e não-medicamentosas que podem ser aplicadas isoladamente ou em conjunto.

O DM 2 é um distúrbio crônico, complexo e de difícil tratamento ao longo do tempo. De modo geral, a maior parte desses sujeitos está com excesso de peso, ou ainda são hipertensos. Desse modo, são incapazes de permanecerem próximos de uma normoglicemia sem a adoção de Antidiabéticos Orais (AOs).,2, Assim, embora outros fatores tenham relevância, o cumprimento da terapia com hipoglicêmicos é uma estratégia chave no controle metabólico desta doença, ao longo do tempo. ${ }^{5-6}$

A despeito disto, a literatura científica evidencia que a adesão à terapia farmacológica entre pessoas com diabetes é de $67,5 \%$, considerada a mais baixa quando comparada à adesão a outros aspectos do tratamento dessa doença. ${ }^{7}$ Nos Estados Unidos, por exemplo, uma revisão sistemática verificou que a adesão ao tratamento farmacológico com AOs variou de 36 a $93 \% .{ }^{8}$ No México foi encontrado um percentual equivalente a $58 \%$ de adesão ao uso de AOs. ${ }^{9}$ Na Europa há publicações, na Espanha e Portugal, que detectaram percentuais situados entre 15,6 e $78 \%$ e entre 60 e $76 \%$, respectivamente, de cumprimento à terapia medicamentosa em sujeitos com DM 2.5,10

Ao não cumprir o regime medicamentoso com AOs o paciente com DM 2 não obtém os benefícios fisiológicos esperados (normoglicemia), deteriora a relação paciente/profissional de saúde, além de elevar os custos médicos com a gênese de complicações micro e macrovasculares, como infarto agudo do miocárdio, acidente vascular encefálico, insuficiência renal, retinopatias, amputações e óbitos diretos. ${ }^{2,11}$

É consenso que o termo adherence avalia a cooperação do paciente ao tratamento de forma mais abrangente, contemplando o estilo de vida dos sujeitos e a sua interação com o profissional de saúde, enquanto o termo compliance fica restrito à obediência dos pacientes a tomada de medicamentos. Entretanto, entre os acometidos por DM 2, a tomada correta dos AOs é a principal estratégia para o alcance da normoglicemia. ${ }^{2,10}$ Mesmo com a alusão destas informações, é sabido que ainda não é rotina na prática clínica da atenção primária de saúde avaliar o cumprimento farmacológico dos sujeitos com DM 2, seja através de métodos diretos e/ou indiretos. ${ }^{12}$

O desenvolvimento de pesquisas sobre adesão medicamentosa em sujeitos com DM 2, por parte de enfermeiros, no Brasil, ainda é tímido. Prova disto é que uma revisão de literatura sobre a adesão do portador de DM 2 ao tratamento com AOs analisou 27 estudos nacionais e internacionais e constatou o predomínio das publicações estrangeiras $(81,4 \%)$ e uma menor produção dos enfermeiros $(26 \%)$ em relação aos demais profissionais de saúde. Tendo em vista a amplitude do problema da adesão ao tratamento medicamentoso do DM 2 acredita-se ser relevante que os enfermeiros realizem pesquisas inéditas que revertam essa lacuna na literatura. ${ }^{6}$ Interessa, portanto, neste artigo, avaliar o cumprimento do tratamento farmacológico entre usuários de AOs atendidos na atenção primária da cidade de Fortaleza-Ceará, Brasil.

\section{MÉTODO}

\section{Tipo de estudo e amostra}

Trata-se de um estudo transversal realizado no período entre janeiro a julho de 2009, em 12 centros de saúde da família de Fortaleza. Essa metrópole do nordeste brasileiro é dividida em seis regiões geoeconômicas e administrativas e possuía, em 2009, 173.000 diabéticos cadastrados na atenção básica de saúde. Para obter dados representativos da adesão ao tratamento com $\mathrm{AO}$ nesta cidade, escolheram-se, de cada região, dois serviços de atenção em DM 2, localizados em bairros distintos no que diz respeito à infraestrutura, serviços e situação socioeconômica dos moradores.

A amostra foi distribuída por conglomerados e, para o cálculo, utilizou-se uma fórmula para populações infinitas. A variável "não cumprimento da terapia farmacológica pelos diabéticos da atenção básica" foi escolhida como desfecho e sua prevalência adotada foi de $60 \% .{ }^{6} \mathrm{O}$ nível de significância e o erro amostral foram $a=0,05$ e $5 \%$ respectivamente. O tamanho da amostra ficou estabelecido em 369 diabéticos. Considerando uma taxa de $10 \%$ de perdas na qual foram incluídas recusas; hospitalizações; desistências; informações erradas e/ ou não disponíveis, o tamanho amostral culminou em 406. Contudo, atendendo a alguns usuários que solicitaram a participação, ampliou-se a amostra para 437 sujeitos.

\section{Seleção dos sujeitos}

Os sujeitos da pesquisa foram selecionados, dentro de cada serviço de saúde escolhido, de 
maneira não-probabilística, desde que atendessem aos seguintes critérios de inclusão: ter diagnóstico confirmado de DM 2 registrado no prontuário ou ficha de saúde; ser atendido(a) nos serviços da rede de atenção primária selecionados para o estudo; residir em Fortaleza e ter telefone para contato; estar em tratamento com AO; e estar há pelo menos seis meses, com a mesma prescrição, a fim de evitar mudanças na terapêutica medicamentosa que possam interferir no estudo e ter prontuário ou ficha de saúde disponível no serviço. Os critérios de exclusão estabelecidos foram: estar em tratamento combinado com AOs e insulina; ficar hospitalizado no período da coleta de dados; e ser dependente de outras pessoas para tomar os AOs.

\section{Teste piloto}

Após a aprovação do projeto pelo Comitê de Ética em Pesquisa em Seres Humanos da Universidade Federal do Ceará, sob o protocolo $n^{\circ}$ 44/07, retornou-se a uma das unidades de saúde selecionadas para dar início ao teste piloto. Antes, porém, os enfermeiros responsáveis pela coleta de dados participaram de um treinamento de 16 horas para assegurar a fidedignidade dos dados. $\mathrm{O}$ teste piloto foi realizado com 39 usuários de $\mathrm{AO}$ e as informações colhidas não foram computadas nesta pesquisa.

\section{Coleta de dados}

Os dados foram coletados entre março e julho de 2009 e os sujeitos convidados a participarem enquanto se encontravam na fila da farmácia. $\mathrm{Na}$ ocasião, e mediante o aceite ao convite, colheu-se os dados sociodemográficos, os clínicos, aqueles referentes ao tratamento do DM 2, bem como o endereço completo e o telefone. Posteriormente, foi entregue uma sacola plástica como cortesia e orientou-se para que ali guardassem apenas os AOs recebidos e que a partir daquele dia, mesmo que ainda tivessem algum comprimido em casa, fizessem uso dos AOs acondicionados na sacola. As informações colhidas nessa etapa foram confrontadas com as dos prontuários e/ou fichas de saúde a fim de averiguar a veracidade das mesmas. Em seguida, foram submetidas aos critérios de inclusão e exclusão para a seleção dos elegíveis.

\section{Instrumentos}

Dez dias após a fase supracitada os sujeitos selecionados receberam uma visita surpresa em seus respectivos domicílios. Para justificar a visita foi colocado que se tratava de uma atividade da unidade para averiguar a glicemia capilar dos pesquisados. Mediante o consentimento de cada um, foram aplicados o Teste de Batalla (TB), a Medida de Adesão ao Tratamento (MAT) e o Teste da Contagem de Comprimidos (TCC).

O TB foi adaptado para o DM 2 pelos autores, pois ele inicialmente havia sido criado para hipertensos, quando foi detectado elevada sensibilidade $(85 \%)$ e valor preditivo positivo $(75 \%)$. O referido teste envolveu três perguntas: 1. O DM 2 é uma enfermidade para toda a vida? 2. O DM 2 pode ser controlado com dieta e medicação? 3 . Cite dois ou mais órgãos que podem ser atingidos pelo DM 2. Segundo as indicações dos criadores do TB, quando os sujeitos respondem corretamente a todas as perguntas, eles são classificados como cumpridores do tratamento medicamentoso. ${ }^{13}$

A MAT é um teste com boa consistência interna (Alpha de Cronbach $=0,66$ ) estruturado a partir da fusão das questões propostas por Morisk, Green e Levine e Delgado \& Lima. ${ }^{10}$ Os sujeitos responderam a este teste de forma dicotômica, isto é, sim ou não às seguintes indagações: 1 . Alguma vez esqueceu-se de tomar os AOs? 2. Alguma vez foi descuidado com a hora de tomar os AOs? 3. Alguma vez deixou de tomar os AOs por ter se sentido melhor? 4. Alguma vez deixou de tomar os AOs, ou por sua iniciativa, ou por ter se sentido pior? 5 . Alguma vez tomou mais de um ou vários $\mathrm{AOs}$, ou por iniciativa própria, ou por ter se sentido pior? 6 . Alguma vez interrompeu o tratamento com os AOs por ter deixado acabar o medicamento? Foram considerados cumpridores os sujeitos que responderam "não" a todos os itens perguntados. ${ }^{10}$

Durante a visita domiciliária, antes de proceder ao TCC, foi mensurada a glicemia capilar, mediante a utilização de glicosímetro e da respectiva fita-teste da marca Optium ${ }^{\circledR}$. A mensuração da glicemia capilar foi realizada para justificar a visita domiciliária surpresa, orientando-se que se tratava de uma atividade da unidade básica de saúde. Os valores detectados levou em consideração o estado de jejum ou pós-prandial dos sujeitos. Dessa maneira, os resultados foram interpretados da seguinte forma naqueles em jejum: 70-110 mg/dL (Bom), 111-140 mg/dL (Aceitável) e > 140 mg/Dl (Insatisfatório). Já nos sujeitos alimentados, a classificação foi a seguinte: 70-140 mg/Dl (Bom), 141-160 mg/ dL (Aceitável) e > $160 \mathrm{mg} / \mathrm{dL}$ (Insatisfatório). Entretanto, para fins estatísticos, os valores da glicemia capilar classificados como bom e aqueles com valor 
aceitável ou insatisfatório foram denominados normal e alterado, respectivamente. ${ }^{14}$

O cumprimento medicamentoso através do TCC foi calculado a partir do percentual obtido entre a razão do número de $\mathrm{AOs}$ tomados e o número de AOs prescritos. Os percentuais foram interpretados como seguem: entre $80 \%-110 \%$, Bom cumpridor; $<80 \%$, Hipocumpridor; $>110 \%$, Hipercumpridor. Os Hipocumpridores e os Hipercumpridores foram classificados com não cumpridores da terapia farmacológica. ${ }^{15}$

De modo gentil, os pesquisadores solicitaram aos sujeitos que trouxessem a sacola plástica com as respectivas cartelas de AOs, além da prescrição médica correspondente ao dia em que receberam a sacola plástica. Em alguns casos o TCC foi difícil, pois constatou-se que, ou as cartelas guardadas na sacola plástica estavam intactas, ou foram utilizadas de forma alternada com outras cartelas recebidas antes da participação na pesquisa. Nestas situações, com o auxílio da prescrição médica, contou-se os comprimidos tomados a partir das cartelas que haviam sido utilizadas.

Em 14 sujeitos não foi possível efetuar o TCC, pois 12 referiram ter abandonado o tratamento e dois alegaram a falta dos medicamentos. Ao serem questionados sobre tal fato, constatou-se que tinham cedido para vizinhos ou parentes. Desse modo, foram considerados não cumpridores do tratamento enfocado no presente estudo.

Frente à densidade dos dados encontrados no estudo piloto, outro recurso metodológico utilizado foi o diário de pesquisa de campo; fato que consistiu no registro manual de intercorrências e atitudes inadequadas dos investigados acerca da tomada e/ou armazenamento dos AOs, do estilo de vida e do tratamento do DM 2. Dessa maneira, durante todas as visitas domiciliárias, os pesquisadores realizaram apontamentos com base nos relatos dos sujeitos com DM 2 e na sua observação do ambiente.

\section{Análise dos dados}

Os dados sofreram tripla digitação e foram armazenados em um banco no Programa Excel. O processamento das informações ocorreu no software SPSS versão 17.0. Fatores considerados componentes do controle metabólico do DM 2 como estado nutricional, sedentarismo, glicemia entre outras foram associadas com o cumprimento medicamentoso, através dos resultados do TCC. Para tal fim, foi empregado o teste exato de Fisher.

\section{RESULTADOS}

Os resultados mostraram que houve uma maior participação de sujeitos do sexo feminino $(70,3 \%)$, brancos $(48,1 \%)$ e pardos $(34,8 \%)$. Quanto à faixa etária foram encontrados usuários entre 18 e 92 anos de idade, distribuídos nas seguintes faixas etárias: $18-59$ anos $(41,1 \%), 60-69$ anos $(29,5 \%)$, $70-79$ anos $(20,1 \%)$ e $80-92$ anos (9,3\%). A média de idade e de anos de estudo foi de 63,1 anos $(\mathrm{DP} \pm 11,6)$ e 4,3 anos $(\mathrm{DP} \pm 3,7)$, respectivamente. Um pouco mais da metade $(55,8 \%)$ dos investigados era casado(a) ou vivia numa relação estável consensual, enquanto uma minoria $(6,9 \%)$ era solteiro(a). Parcela substancial tinha casa própria pessoal $(84,9 \%)$ e cerca da metade dos estudados $(45,8 \%)$ vivia numa família nuclear composta por companheiro(a), filhos e/ ou netos. A maioria era aposentada $(49,4 \%)$ e esta fonte de renda constituía a principal forma de sustentação da prole dos sujeitos $(48,7 \%)$. Houve um predomínio das classes econômicas D e C. A média da renda familiar mensal era de U\$ 445,9 dólares (DP $\pm 327,1)$.

Os resultados obtidos a partir da aplicação do TB permitiram evidenciar que boa parte dos diabéticos entrevistados $(73,2 \%)$ tinha conhecimento da cronicidade e da inexistência de cura do DM 2, e 87,9\% deles entendiam que a doença pode ser controlada com dieta e medicamentos (Tabela 1).

Tabela 1 - Distribuição dos usuários com DM 2 em uso de AO segundo os resultados do Teste de Batalla. Fortaleza-CE, 2009

\begin{tabular}{lrrr}
\hline Perguntas & $\mathbf{n}$ & \% & IC \\
\hline O DM 2 é uma enfermidade & & & \\
para toda vida? & 320 & 73,2 & $70,7-79,1$ \\
Sim & 106 & 24,3 & $20,9-29,3$ \\
Não & 11 & 2,5 & $1,9-5,5$ \\
Não sabe & & & \\
O DM 2 pode ser controlado & & \\
com dieta e medicamento? & 384 & 87,9 & $86,1-92,2$ \\
Sim & 45 & 10,3 & $7,8-13,9$ \\
Não & 6 & 1,4 & $0,6-6,6$ \\
Não sabe & & & \\
Citar $\geq 2$ órgãos afetados pelo & & \\
DM 2 & 206 & 47,2 & $41,9-53,5$ \\
Nenhum acerto & 88 & 20,1 & $16,5-24,3$ \\
1 acerto & 143 & 32,8 & $28,0-38,7$ \\
$\geq 2$ acertos & & & \\
Resultado do Teste de Batalla & 111 & 25,4 & $21,4-29,8$ \\
Cumpridor & 326 & 74,6 & $70,2-78,6$ \\
Não cumpridor & & & \\
\hline
\end{tabular}


A partir do teste MAT, ficou evidente que a maioria dos investigados já esqueceu de tomar os AOs $(54 \%)$ ou pelo menos tomou num horário diferente $(64,1 \%)$ do determinado pela prescrição médica. Além disso, 82,4\% dos sujeitos negaram já ter deixado de tomar $\mathrm{AOs}$ devido à sensação de melhora ou de piora do DM 2. Um percentual elevado $(82,8 \%)$ também afirmou nunca infringir, para mais ou menos, a dose de AOs estabelecida por conta própria, por sentir piora do DM 2 (Tabela 2).

Tabela 2 - Distribuição dos usuários com DM 2 em uso de AO segundo Teste MAT. FortalezaCE, 2009

\begin{tabular}{lrrr}
\hline Perguntas & $\mathbf{n}$ & $\%$ & IC-95\% \\
\hline Já se esqueceu de tomar os & & & \\
AOs & 236 & 54,0 & $49,2-58,7$ \\
Sim & 201 & 46,0 & $41,3-50,8$ \\
Não & & \\
Já foi descuidado com o horá- & & \\
rio de tomar os AOs & 280 & 64,1 & $59,4-68,5$ \\
Sim & 157 & 35,9 & $31,5-40,6$ \\
Não & & \\
Já deixou de tomar os AOs & & \\
por sentir-se melhor & 77 & 17,6 & $14,2-21,6$ \\
Sim & 360 & 82,4 & $78,4-85,8$ \\
Não & & \\
Já deixou de tomar os AOs & & \\
por sentir-se pior & 77 & 17,6 & $14,1-21,4$ \\
Sim & 360 & 82,4 & $78,6-85,9$ \\
Não & & \\
Já tomou mais de um ou vá- \\
rios AOs, por sua iniciativa \\
por sentir-se pior & & \\
Sim & 75 & 17,2 & $13,8-21,1$ \\
Não & 362 & 82,8 & $78,9-86,2$ \\
Já interrompeu o tratamento & & \\
por ter deixado acabar os AOs & & \\
Sim & 166 & 38,0 & $33,4-42,7$ \\
Não & 271 & 62,0 & $57,3-66,6$ \\
Resultado do MAT & & \\
Cumpridor & 60 & 13,7 & $10,7-17,4$ \\
Não cumpridor & 377 & 86,3 & $82,6-89,3$ \\
\hline & &
\end{tabular}

Apesar dos achados do teste MAT apontarem que $62 \%$ dos entrevistados nunca interromperam a terapêutica medicamentosa do DM 2, por falta de $\mathrm{AO}$, ao se consultar os registros do diário de campo adotado durante a visita domiciliar foi constatado que $10,6 \%$ pararam o tratamento por falta de AO. Por sua vez, os dados do diário de campo revelaram ainda algumas estratégias incorretas dos sujeitos para manter o número de comprimidos necessários para sua terapêutica do
DM 2, a saber: $11,5 \%$ compartilham cartelas de AO com outros doentes e $9,7 \%$, especificamente, com seu cônjuge, também acometido pelo DM 2.

O TCC apontou que $1,8 \%, 37,6 \%$ e $60,4 \%$ eram Hipercumpridor, Bom cumpridor e $\mathrm{Hi}$ pocumpridor à terapia com AO. Assim, 61,2\%, ( $\mathrm{IC}=54,7-67,3 \%$ ) eram não cumpridores da terapia medicamentosa para o DM2. Em média, o número de drágeas ao dia e o percentual desse cumprimento eram de $3,0(\mathrm{DP} \pm 1,7)$ e $71,3 \%(\mathrm{DP} \pm 32,5)$, respectivamente. Os principais AOs usados eram metformina $(22,9 \%)$, glibenclamida $(21,5 \%)$ e a associação destas (43,5\%).

Aproximadamente $95 \%$ e $76 \%$ dos sujeitos não cumpridores do tratamento com AOs já faltaram às consultas do programa terapêutico para DM $2(p=0,000)$ e estão com a glicemia acima dos valores recomendados $(p=0,013)$, respectivamente (Tabela 3).

Tabela 3 - Associação entre o cumprimento da terapia medicamentosa com antidiabéticos e componentes do controle metabólico do DM 2. Fortaleza-CE, 2009

\begin{tabular}{|c|c|c|c|c|c|}
\hline \multirow[t]{2}{*}{ Variáveis } & \multicolumn{2}{|c|}{$\begin{array}{l}\text { Cumpri- } \\
\text { dor* }^{*}\end{array}$} & \multicolumn{2}{|c|}{$\begin{array}{l}\text { Não cum- } \\
\text { pridor* }\end{array}$} & \multirow[t]{2}{*}{$\mathrm{p \dagger}$} \\
\hline & $\mathrm{n}$ & $\%$ & $\mathrm{n}$ & $\%$ & \\
\hline $\begin{array}{l}\text { Já faltou às consultas } \\
\text { para DM } 2\end{array}$ & & & & & 0,000 \\
\hline Sim & 9 & 5,9 & 144 & 94,1 & \\
\hline Não & 51 & 18,3 & 228 & 81,7 & \\
\hline $\begin{array}{l}\text { Possui comorbidades } \\
\text { associadas }\end{array}$ & & & & & 0,178 \\
\hline Sim & 53 & 14,8 & 306 & 85,2 & \\
\hline Não & 7 & 9,0 & 71 & 91,0 & \\
\hline $\begin{array}{l}\text { Pratica atividade física } \\
\text { regularmente }\end{array}$ & & & & & 0,373 \\
\hline Sim & 13 & 21,7 & 93 & 78,3 & \\
\hline Não & 47 & 24,7 & 284 & 75,3 & \\
\hline Glicemia está normal & & & & & 0,013 \\
\hline Sim & 23 & 38,3 & 37 & 23,6 & \\
\hline Não & 89 & 61,7 & 288 & 76,4 & \\
\hline $\begin{array}{l}\text { Está com excesso de } \\
\text { peso }\end{array}$ & & & & & 0,264 \\
\hline Sim & 18 & 33,3 & 36 & 66,7 & \\
\hline Não & 98 & 28,2 & 250 & 71,8 & \\
\hline
\end{tabular}

O diário de pesquisa de campo, utilizado nas visitas domiciliárias, permitiu identificar também os seguintes problemas na tomada dos AOs, independente dos resultados dos métodos de 
adesão, a saber: $26,5 \%$ tinham um grande número de comprimidos para prescrição (ou seja, deixaram de tomá-los conforme prescrito) e 10,6\% usavam cartelas com drágeas violadas, em alguns casos com prazo de validade expirado, e abandonaram o tratamento, respectivamente. Uma menor parte, $9,7 \%$, usavam os antidiabéticos apenas quando se lembravam e 6,2\% tomavam menos que o indicado devido confusão com a dose prescrita.

\section{DISCUSSÃO}

O TB identificou que aproximadamente $75 \%$ dos pesquisados não cumpriam a terapia medicamentosa para DM 2. Fora isso, este método evidenciou que os sujeitos tinham um baixo conhecimento sobre a doença, pois praticamente metade dos pesquisados $(47,2 \%)$ não soube mencionar um órgão que fosse afetado pelo DM 2. Os poucos estudos brasileiros encontrados que aplicaram este método com diabéticos dectaram prevalências de não cumprimento farmacológico menores com valores de $10 \%$, 32\% e $67 \% .{ }^{16-18}$ Contudo, vale ressaltar que as amostras destas pesquisas eram pequenas, portanto, mais susceptíveis a percentuais distorcidos. $\mathrm{O}$ estudo que mais se assemelhou aos nossos resultados foi um hispânico, o qual detectou que $84,4 \%$ dos investigados não cumpriam a terapia medicamentosa. ${ }^{6}$

$\mathrm{O}$ TB, além de evidenciar a falta de conhecimento do doente a respeito da sua patologia, pôde levantar outro problema: a assimilação das informações sobre DM 2 dos profissionais da saúde, pelos clientes.

O caráter crônico da DM 2 exige um acompanhamento contínuo pelos profissionais de saúde e demanda que os sujeitos tenham respostas corretas para as suas dúvidas acerca do manejo clínico desta patologia. Isto possibilita uma maior credibilidade em torno dos profissionais e, consequentemente, acarreta num baixo grau de incerteza terapêutica, pois na ausência ou má qualidade da informação, o cliente diabético não possui parâmetros para um autocuidado. ${ }^{19} \mathrm{~A}$ falta de compreensão acerca dos AOs vem se constituindo numa das causas de insegurança do cliente, podendo desencadear atos inconsequentes, como a recomendação do próprio medicamento a outros indivíduos ou até mesmo abandono do tratamento, ambos detectados neste trabalho. ${ }^{20}$ A literatura aponta ainda que estes comportamentos e a incompreensão do cliente acerca da tomada de medicamentos possam estar relacionados com o fato do quadro de representações sociais dos sujeitos estarem mais próximos dos sujeitos da comunidade do que dos

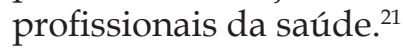

Em relação ao Teste MAT, foi encontrada apenas uma publicação com sujeitos com DM 2, quando a prevalência de não cumprimento medicamentoso foi de apenas $21,7 \% .{ }^{22}$ Em publicações nacionais e internacionais que utilizaram o método MAT, precursor do MAT, também se verificou menores valores para o desfecho em questão, conforme as prevalências de $20 \%, 46 \%, 50 \%, 58,1 \%$ e $68,8 \%$.9-10,16-17,23-24 Foi identificado apenas um estudo desenvolvido nos Estados Unidos que concluiu que $87 \%$ dos diabéticos eram não cumpridores do tratamento medicamentoso..$^{25}$

O TCC foi, dentre os três critérios de cumprimento farmacológico, o que menos detectou casos de não cumpridor $(61,2 \%)$, em detrimento do Teste MAT $(86,3)$ e do TB $(74,6 \%)$. Ao analisar a literatura em diversas bases de dados como Bireme, PubMed e Scopus foi constatado uma carência de publicações desenvolvidas com brasileiros sobre adesão ao uso de AOs/anti-hipertensivos que adotassem este critério em suas metodologias. Ao fim, apenas dois estudos foram encontrados; o primeiro detectou uma prevalência de não adesão de $61,6 \%$ e o segundo detectou que $34 \%$ dos diabéticos avaliados não eram aderentes. ${ }^{26-27}$

No contexto internacional este método é mais utilizado em pesquisas de adesão com sujeitos com DM 2. Em países como Polônia, França, Suíça, Holanda, Grécia, Estados Unidos, Jamaica, Espanha, México e Tailândia, foram encontradas prevalências de não cumprimento farmacológico de 6,5\%, $13 \%, 17,1 \%, 25,2 \%, 21,4 \%, 29 \%, 44 \%, 64,6 \%, 73 \%$ e $93,8 \%$, respectivamente, em diabéticos, a partir da contagem de comprimidos. Os achados da pesquisa ora apresentada foram semelhantes aos encontrados em diabéticos de países em desenvolvimento como Jamaica, Tailândia e México e desenvolvidos como a Espanha. ${ }^{28-34}$

Seja através da avaliação do conhecimento sobre sua doença, do número de comprimidos tomados ou dos comportamentos adotados no cotidiano na tomada destes, o cumprimento do tratamento farmacológico do DM 2 é baixo e certamente repercutirá no desenvolvimento precoce de complicações micro e macrovasculares nesses sujeitos.

Em termos globais, independente do critério adotado, a prevalência de não adesão ao tratamento com AOs no Brasil é ainda desconhecida. Entretanto, pesquisa multicêntrica desenvolvida em nove países da América Latina constatou que 60,1\% dos sujeitos com DM 2 são não cumprido- 
res da terapêutica medicamentosa com AOs..$^{35} \mathrm{Ao}$ se levar em consideração que o Brasil foi um dos avaliados deste estudo e que não há fatos que atenuem esta problemática no país, é provável que esse valor seja um parâmetro da atual condição deste problema no Brasil.

Frente a este quadro, acreditamos que, uma medida viável na atenção primária para melhorar a adesão medicamentosa do sujeito com DM 2 seria a realização de visitas domiciliares sistemáticas àqueles clientes com controle glicêmico inadequado ou ainda daqueles classificados como não aderentes, ainda nas unidades básicas de saúde, a fim de aplicar o processo de educação em saúde, ma vez que conhecer o meio ambiente em que vivem as pessoas com diabetes é muito importante, pois pode revelar fatores positivos e negativos no enfrentamento da doença.

Podemos constatar neste estudo, durante a execução das visitas ao domicílio dos clientes com DM 2, que alguns aspectos importantes do meio, como a escolaridade, a profissão, o nível econômico, as relações familiares, os grupos sociais de apoio, o estresse, entre outros, guardavam relações diretas e indiretas com a tomada dos AOs.

Além desses benefícios, o ato de visitar o domicílio dos pacientes com DM 2 pode ajudar a encorajar muitos desses sujeitos a afirmar a sua não adesão ao tratamento e suas causas, através do diálogo e da observação. Dessa forma, acreditamos que uma combinação de métodos indiretos de adesão e visita domiciliária seria bastante exitosa no combate a problemática da tomada dos AOs, pois o primeiro detectaria sujeitos não cumpridores e o segundo seria veículo para a aplicação da educação em saúde e empoderamento do sujeito com DM 2 e, consequentemente, elevaria o cumprimento da terapia com $\mathrm{AO}$.

Especialmente no quesito educação em saúde, a prática sistemática de visitas ao domicílio dos sujeitos com DM 2, seria importante para reverter o fato de menos de $20 \%$ dos clientes com DM 2 terem acesso a essa modalidade terapêutica na América Latina, ${ }^{35}$ o que, consequentemente, levaria ao alcance de uma atenção integrada ao sujeito com diabetes. ${ }^{36}$

\section{CONCLUSÕES}

Uma das potencialidades desta pesquisa reside na representatividade, tanto em termos amostrais como geográficos, abarcando todas as seis regiões que compõem a cidade de Fortaleza e na utilização de métodos de investigação, como a contagem de comprimidos, pouco usado em investigações com clientes com DM 2 no Brasil. Dentre as suas limitações é possível apontar a adoção de métodos indiretos que apesar de válidos, não são perfeitos, e necessitam de treinamento intensivo dos pesquisadores, a fim de minimizar possíveis problemas.

Como implicações, é notável o fato de que conhecida a prevalência do não cumprimento do regime medicamentoso com $\mathrm{AO}$, os profissionais de saúde da rede básica de saúde, especialmente os enfermeiros, terão subsídios para conhecer esses clientes e planejar e implementar ações educativas baseadas nas suas necessidades reais. No futuro, acreditamos que outros estudos poderão ser planejados na perspectiva de conhecer diagnósticos de enfermagem e, ainda, explorar e/ou conhecer os fatores que estão envolvidos nesta não adesão, suas nuances e possíveis focos para o direcionamento de cuidados de enfermagem.

A expansão e desenvolvimento do Programa de Saúde da Família (PSF) aumentou a disponibilização dos AOs e até de insulina, para o tratamento do DM 2. Assim, a disponibilidade de AO não parece ser o ponto central do problema do não cumprimento medicamentoso, já que o SUS oferta os fármacos a todos os doentes cadastrados no programa Hiperdia, mesmo com alguns dias de atrasos.

A causa dos problemas em relação à tomada dos AOs talvez esteja na motivação do cliente com DM 2 e do profissional de saúde ou ainda na estrutura dos serviços da atenção primária. O cliente precisa compreender que a adesão ao tratamento medicamentoso caminha, simultaneamente, com a prevenção de complicações crônicas do DM 2, maior expectativa de vida, manutenção da sua independência e qualidade de vida. Por sua vez, os profissionais de saúde necessitam de melhores condições laborais, como uma maior disponibilidade de profissionais em suas equipes, ou ainda, a redução de famílias atendidas, a fim de que estratégias como a visita domiciliar e a educação em saúde, propriamente dita, possam alcançar um maior número de clientes com DM 2 e não apenas aqueles que já apresentam complicações vasculares. Por fim, o resgate dos fundamentos da promoção da saúde também necessita permear as ações dos profissionais da rede básica, ou seja, a relação com o cliente com DM 2 deve ser horizontal e de capacitação, processo que pode envolver a família, a comunidade e a equipe multiprofissional. 


\section{REFERÊNCIAS}

1. United Kingdom Prospective Diabetes Study Group (UKPDS). Intensive blood-glucose control with sulphonylureas or insulin compared with conventional treatment and risk of complications in patients with type 2 diabetes (UKPDS33). Lancet. 1998 Sep; 352(9131):837-53.

2. Skyler JA. Relação do controle glicêmico com as complicações diabeticas. In: Inzucchi, S. Diabete Melito: manual de cuidados essenciais. Porto Alegre (RS): Artmed; 2007. p.334-47.

3. Rozenfeld Y, Hunt JS, Plauschinat C, Wong KS. Oral antidiabetic medication adherence and glycemic control in managed care. Am J Manag Care. 2008 Feb; 14(2):71-5.

4. Krentz AJ, Bailey CJ. Oral antidiabetic agents: current role in type 2 diabetes mellitus. Drugs. 2005 Jan; 65(3):385-411.

5. García-Pérez AM, Leiva Fernandez F, Martos Crespo F, García Ruiz AJ, Prados Torres D, Sánchez C, et al ¿Como diagnosticar el cumplimiento terapêutico en atención primaria? Med Família (And). 2000 Jun; 1(1):13-9.

6. Araújo MFM, Damasceno MMC. La adhesión del diabético al tratamiento farmacológico com hipoglicemiantes orales: uma investigación bibliográfica. Rev Enferm Int. 2008 Out-Dez; 84(4):26-31.

7. Dimatteo MRP. Variations in patient's adherence to medical recommendations: a quantitative review of 50 years of research. Med Care. 2004 Mar; 42(3): 200-9.

8. Cramer JA. A systematic review of adherence with medications for diabetes. Diab Care. 2004 May; 27(5):1218-24.

9. Villarreal-Ríos E, Paredes-Chaparro A, MartinezGonzaléz L, Galicia-Rodriguez L, Vargas-Daza E, Garza-Elizondo ME. Control de los pacientes con diabetes tratados sólo con esquema farmacológico. Rev Med Inst Mex Seguro Soc. 2006 Nov; 44(4):303-8.

10. Delgado AB, Lima ML. Contributo para a validação concorrente de uma medida de adesão aos tratamentos. Psicol Saúde Doenças. 2001 Nov; 2(2):81-100.

11. Capriotti T. Type 2 diabetes epidemic increases use of oral anti-diabetic agents. MEDSURG Nursing. 2005 Oct; 14(5):341-7.

12. Pladevall M, Williams K, Potts LA, Divine G, Xi $\mathrm{H}$, Lafata JE. Clinical outcomes and adherence to medications measured by claims data in patients with diabetes. Diab Care. 2004 Dec; 27(12):2800-5.

13. Batalla C, Blanquer A, Ciurana R, Garcia M, Cases E, Pérez A, et al. Cumplimiento de la prescripción farmacológica en pacientes hipertensos. Atenc Prim. 1994 Ene; 11:185-91.

14. Pascali PM. Monitorização da glicemia. In: Grossi, SAA, Pascali PM, organizadores. Cuidados de enfermagem em diabetes mellitus. São Paulo: Sociedade Brasileira de Diabetes; 2009. p.41-55.
15. Sackett DL, Haynes RB, Gibson ES. Randomised clinical trial of strategies for improving medicacion compliance in primary hypertension. Lancet. 1975 May; 1(7918):1205-7.

16. Bezerra DS, Silva AS, Carvalho ALM. Avaliação das características dos usuários com hipertensão arterial e/ou diabetes mellitus em uma Unidade de Saúde Pública, no município de Jaboatão dos Guararapes-PE, Brasil. Rev Ciênc Farm Básica 2009 Jan-Abr; 30(1):57-61.

17. Alencar EKF. Avaliação do nível de adesão à terapêutica medicamentosa: estudo com diabéticos em centro de saúde da família em Fortaleza-CE [Monografia]. Fortaleza (CE): Faculdade Grande Fortaleza, 2009.

18. Martins CB, Parolina RD, Caravante Junior FP, Farhat FCLG, Carmo TA. Melhoria da Adesão ao tratamento medicamentoso de portadores de diabetes mellitus e hipertensão arterial por meio da atenção farmacêutica. In: Anais da $5^{\mathrm{a}}$ Mostra Acadêmica e $9^{\circ}$ Seminário de extensão UNIMEP, 2007; Piracicaba-Brasil. Piracicaba(SP): UNIMEP; 2007.

19. Apóstolo JLA, Viveiros CSC, Nunes HIR, Domingues HRF. Incerteza na doença e motivação para o tratamento em diabéticos tipo 2. Rev Latino-am Enfermagem. 2007 Jul-Ago; 15(4):575-82.

20. Gimenes HT, Zanetti ML, Otero LM, Teixeira CRS. O conhecimento do paciente diabético tipo 2 acerca dos antidiabéticos orais. Ciênc Cuidado Saúde. 2006 Set-Dez; 5(3):317-25.

21. Leite SN, Vasconcelos MPC. Adesão a terapêutica medicamentosa: elementos para discussão de e pressupostos adotados na literatura. Ciênc Saúde Coletiva. 2003 Jul-Set; 8 (3):775-82.

22. Gimenes HT, Zanetti ML, Haas VJ. Fatores relacionados com à adesão do paciente diabético à terapêutica medicamentosa. Rev Latino-am Enfermagem. 2009 Jan-Fev; 17(1):46-51.

23. Yurgin NR, Boye KS, Dilla T, Suriñach NL, Llach $X B$. Physician and patient management of type 2 diabetes and factors related to glycemic control in Spain. Patient Preference Adherence. 2008 Feb; 2: 87-95.

24. Prado Junior JC, Kupek E, Mion D. Validity of four indirect methods to measure adherence in primary care hypertensives. J Hum Hypertens. 2007 Jul; 21(7):579-84.

25. Krapek K, King K, Warren SS, George KG, Caputo DA, Mihelich K, et al. Medication adherence and associeted hemoglobin $\mathrm{A}_{1 \mathrm{c}}$ in type 2 diabetes. Ann Pharmacother. 2004 Sep; 38(9):1357-62.

26. Teixeira ACA. Adesão ao tratamento farmacológico da hipertensão arterial e seus determinantes em pacientes de ambulatório [dissertação]. Fortaleza (CE): Faculdade de Medicina da Universidade Federal do Ceará, Fortaleza, 1998. 
27. Pires GB, Freitas IV. Prevalência de adesão ao tratamento farmacológico hipoglicemiante. Sitientibus. 2006 Jan-Jun; 34:37-45.

28. Guillasseau PJ. Impacto of compliance with oral antihyperglicemic agents on health outcomes in type 2 diabetes mellitus. Treat Endocrinol. 2005 Dez; 4(3):167-75.

29. Giannopoulos S, Kosmidou M, Sarmas I, Markoula S, Pelidou SH, Lagos G, et al. Patient compliance with SSRIs and gabapetin in painful diabetic neuropathy. Clin J Paim. 2007 Mar-Apr; 23(3):267-9.

30. Walker EA, Molitch M, Kramer K, Kahn S, Edelstein S, Smith K, et al. Adherence to preventive medications: predictors and outcomes in the diabetes prevention program. Diab Care. 2006 Sep; 29(9):1997-2002.

31. Swaby P, Wilson E, Swaby S, Pierre R. Chronic disease control and compliance--the HOPE worldwide Jamaica experience. West Indian Med J. 2001 Mar; 50 (Suppl 1):51-3.

32. Mateo JF, Gil-Gullén VF, Mateo E, Orozoco D, Carbayo JA, Merino J. Multifactorial approach and adherence to prescribed oral medications in patients with type 2 diabetes. Int J Clin Pract. 2006 Apr; 60(4):422-8.

33. Prado-Aguilar CA, Martínez YV, Segovia-Bernal Y, Reyes R, Arias-Ulloa A. Performance of two questionnaires to measure treatment adherence in patients with Type-2 Diabetes. BMC Public Health. 2009 Jan; 9(38):1-8.

34. Phumipamorn S, Pongwecharak J, Soorapan S, Pattharachayakul S. Effects of the pharmacist's input on glycaemic control and cardiovascular risks in Mulsim diabetes. Prim Care Diabetes. 2008 Feb; 2(1):31-7.

35. Stewart GL, Tambascia M, Guzmán JR, Etchegoyen F, Carrión JO, Artemenko S. Control of type 2 diabetes in private practice in nine countries of Latin America. Rev Panam Salud Publica. 2007 Jul; 22(1):12-20.

36. Fragoso LVC, Araújo MFM, Lima AKG, Freitas RWJF, Damasceno MMC. Vivências cotidianas de adolescentes com diabetes mellitus tipo 1. Texto Contexto Enferm. 2010 Jul-Set; 19(3):443-51. 